\title{
Radio wave scattering in the outer heliosphere: Preliminary calculations
}

Iver H. Cairns

Department of Physics and Astronomy, University of lowa, Iowa City

\begin{abstract}
Detailed first estimates are presented of angular broadening in the outer heliosphere due to scattering of radio waves by density irregularities: The application is to the $2-3 \mathrm{kHz}$ radiation observed by Voyager. Two plausible turbulence models, which account very well for scattering within $1 \mathrm{AU}$, are extrapolated beyond $10 \mathrm{AU}$. Both models predict significant angular broadening in the outer heliosphere, accounting semiquantitatively alone for the source sizes inferred from roll modulation data. Predictions are presented for radial variations in the apparent source size if scattering is important. Comparisons with available data argue that scattering is important (and indeed is the dominant contributor to the apparent source size) and that the radiation source is located in the outer heliosphere. Other evidence that scattering is important, such as the fluctuations in apparent source direction and intensity, are also identified. The effects of scattering should be included in future analyses of the $2-3 \mathrm{kHz}$ emissions.
\end{abstract}

\section{Introduction}

Density irregularities are well known to scatter radio waves in solar system and astrophysical plasmas, affecting the apparent angular size, intensity, and direction of the radio source, as well as the temporal and spectral variability of the radiation [e.g., Hollweg, 1970; Steinberg et al., 1971, 1985, 1989; Rickett, 1977]. Radiation generated or propagating close to the electron plasma frequency $f_{p}$ is strongly affected by scattering. Indeed, analyses of all known emissions generated near $f_{p}$ and $2 f_{p}$ in the corona and the solar wind within $1 \mathrm{AU}$ show that scattering is very important in interpreting the angular size, directivity and brightness temperature of the radiation. These sources are type III solar radio bursts in the corona and solar wind [Steinberg et al., 1971; $1985]$ and $2 f_{p}$ radiation generated in Earth's foreshock [Lacombe et al., 1988]. Scattering also significantly affects AKR observed in the solar wind [Steinberg et al., 1989] even though it is generated and propagates well above $f_{p}$ from its auroral sources.

The Voyager spacecraft have observed radiation propagating near $2-3.5 \mathrm{kHz}$ in the outer heliosphere [Kurth

Copyright 1995 by the American Geophysical Union.

Paper number 95GL03331

0094-8534/95/95GL-03331\$03.00 et al., 1984; Kurth, 1988; Gurnett et al., 1993; Gurnett and Kurth, 1994] at frequencies $\sim 1-10$ times the local solar wind plasma frequency and $\sim 1-5$ times likely plasma frequencies in the heliosheath. Existing theories all predict generation of the radiation near $f_{p}$ or $2 f_{p}$ in differing outer heliospheric sources [e.g., Cairns et al., 1992; Gurnett et al., 1993]. Modulations of the radiation intensity near $3 \mathrm{kHz}$ during Voyager roll manoeuvers are observed and interpreted in terms of the apparent size of and direction to the radiation source [Kurth, 1988; Gurnett et al., 1993]: near 48 AU these data are consistent with a maximum half-angle $\sim 60^{\circ} \sim 1 \mathrm{rad}$ for the source. It is relevant to note the absence of modulations near $2 \mathrm{kHz}$ and the significant variation in apparent source direction [Gurnett et al., 1993]. In addition, Cairns et al. [1992] ascribed fluctuations in the radiation's intensity and frequency fine structure to scattering and/or ducting. Based upon these properties and the analogies with inner heliospheric radio sources, it is very plausible that scattering off density irregularities might be important in determining the properties of the Voyager $2-3 \mathrm{kHz}$ radiation. This paper provides a first, detailed, semi-quantitative investigation into how scattering affects the source's apparent angular size.

\section{Scattering rates near $50 \mathrm{AU}$ : first calculations}

Two formalisms exist to calculate the angular broadening of sources due to scattering: a geometric optics approach that has been used in most solar system applications [e.g., Hollweg, 1970; Steinberg et al., 1971, 1985, 1989; Lacombe et al., 1988], and the approach based on the parabolic wave equation (hereafter PWE) that is favored in astrophysical applications [e.g., Lee and Jokipii, 1975; Rickett, 1977; Spangler and Armstrong, 1990]. The physical difference is that diffraction is not included in geometric optics. In both cases the apparent half-angle of the source, $\theta_{\mathrm{app}}$, is

$$
\theta_{\mathrm{app}} \approx \sqrt{\theta_{\mathrm{in}}^{2}+\theta_{\mathrm{sc}}^{2}} .
$$

Here, $\theta_{\text {in }}$ and $\theta_{\mathrm{sc}}$ are half-angles for the intrinsic source size and the broadening contribution due to refractive scattering by density irregularities, respectively. In both approaches $\theta_{\mathrm{sc}}$ depends on a path integral $d z$ from the source to the observer of an integrand that depends on the radiation frequency $f$, the local (steady-state) plasma frequency $f_{p 0}(z)$, and the three- 
dimensional wavevector spectrum of density turbulence $P_{n}(\mathbf{k}, z)$. Further shared assumptions are that the inner scale $l_{\mathrm{i}}=q_{\mathrm{i}}^{-1}$ of the turbulence greatly exceeds the radiation's free-space wavelength $\lambda(=c / f)$ and that the outer scale $l_{\circ}$ is much less than the path length $L$.

Geometric optics is conventionally a viable approximation when [e.g., Tatarski, 1971]

$$
L \lesssim l_{\mathrm{i}}^{2} / \lambda
$$

Taking the thermal proton gyroradius as the inner scale $l_{\text {in }}$ and radiation at $2-3 \mathrm{kHz}$, near $50 \mathrm{AU} l_{\text {in }} \sim 10^{6} \mathrm{~m}$, and so by (2) geometric optics is viable only for path lengths $L \lesssim 10^{7} \mathrm{~m} \sim 10^{-4} \mathrm{AU}$. However, the minimum plausible distance to the source is $\sim 50 \mathrm{AU}$ since the Voyager spacecraft are separated by a comparable distance but observe very similar radiation. Geometric optics therefore appears not viable in the outer heliosphere [J.W. Armstrong, personal communication, 1995]. Consequently the PWE formalism is used in this paper.

The turbulence spectrum $P_{n}(\mathbf{k})$ is currently unknown experimentally or theoretically beyond $\sim 5 \mathrm{AU}$. Unanswered questions include how well WKB theory applies and how MHD turbulence driven by interstellar pickup ions affects the spectral index and level of $P_{n}(\mathbf{k})$ at small scales (where scattering is most important). It is therefore necessary to introduce plausible semiquantitative models in order to make first estimates of whether scattering is important in the outer heliosphere. These models must account adequately for observations within $1 \mathrm{AU}$ and be extrapolated plausibly.

For a Gaussian distribution of density irregularities, defined by a characteristic scale $h$ and a fluctuation level $\Delta n / n$, and generalizing the PWE results of Lee and Jokipii [1975] and Rickett [1977] to include finite $f_{\mathrm{po}}(z) / f$, it may be shown that

$\theta_{s c}^{2}(z)=\int d z \frac{2 \sqrt{\pi}}{\left(1-f_{p}(z)^{2} / f^{2}\right)}\left(\frac{f_{p}(z)}{f}\right)^{4} \frac{1}{h(z)}\left(\frac{\Delta n}{n}\right)^{2}$

Scattering thus increases for smaller $h$, and larger ratios $f_{p} / f$ and $\Delta n / n$. Taking $h$ equal to the thermal proton gyroradius $r_{p} \sim 100 \mathrm{~km}$ and $\Delta n / n=10^{-2}$, choices that are both conventional and plausible at $1 \mathrm{AU}$ [e.g., Hollweg, 1970], and writing $\theta_{s c}^{2}=\int d z b^{\prime}\left(f / f_{p 0}, z\right)$, (3) yields $b^{\prime}\left(2 f_{p}\right)=4 \times 10^{-10} \mathrm{rad}^{2} / \mathrm{m}$ at $1 \mathrm{AU}$. This agrees very well with the values $b^{\prime}\left(2 f_{p}\right) \sim 2-6 \times 10^{-10} \mathrm{rad}^{2} / \mathrm{m}$ observed at $1 \mathrm{AU}$ [e.g., Lacombe et al., 1988].

Cautious extrapolation of this model to the outer heliosphere, for the purpose of making first estimates of $\theta_{s c}$ alone (since $P_{n}(\mathbf{k})$ is undoubtedly not Gaussian), is justified by the model's simplicity and the good agreement with observations at $1 \mathrm{AU}$. At $50 \mathrm{AU}$, with $h=r_{p} \sim 10^{3} \mathrm{~km}$ and $\Delta n / n=10^{-2}$ again, the model predicts $b^{\prime}\left(2 f_{p}\right)=2 \times 10^{-11} \mathrm{rad}^{2} / \mathrm{m}$. Then, assuming $b^{\prime}\left(f / f_{p}\right)$ is constant along a $50 \mathrm{AU}$ path length (the minimum likely distance noted above), (3) predicts $\theta_{s c}(f=2 \mathrm{kHz})=2.4 \mathrm{rad}$ and $\theta_{s c}(f=3 \mathrm{kHz})=1.0 \mathrm{rad}$, corresponding to radiation near 4 and 6 times the local $f_{p 0}$, respectively. For reference, a source with plausible dimensions $\sim 1 \mathrm{AU}$ in the outer heliosphere [e.g., Cairns et al., 1992] subtends an intrinsic half-angle $\theta_{\text {in }} \sim 0.01$ $\mathrm{rad}$ at a distance of $50 \mathrm{AU}$. Table 1 compares the scattering results with Gurnett et al.'s [1993] data. Two results follow. First, the apparent source sizes observed are much larger than plausible $\theta_{\text {in }}$ values, suggesting that an effect such as scattering dominates the data. Second, the surprisingly good agreement between model and data makes it plausible that scattering contributes majorly to the observed source size.

Angular broadening measurements of astrophysical sources along lines of sight within $0.3 \mathrm{AU}$ of the Sun yield a second model [Armstrong and Woo, 1980; Spangler and Armstrong, 1990]: the 3-D wavenumber density spectrum is Kolmogorov with a normalization $C_{N}^{2}$ that depends on heliocentric distance $R$; i.e., $P_{n}(\mathbf{k}, R)=$ $C_{N}^{2}(R) k^{-\alpha}$ with $\alpha=11 / 3$ and

$$
C_{N}^{2}(R)=3.9 \times 10^{15}\left(\frac{R}{R_{S}}\right)^{-4.05} \mathrm{~m}^{-20 / 3}
$$

Here $R_{S}$ is the Sun's radius. Since the solar wind speed $v_{s w}$ is independent of $R$ and the background plasma density falls off as $R^{-2}$ in steady-state, this model corresponds to the relative density fluctuations being essentially independent of $R$. For power-law density spectra the angular distribution of the scattered radiation is no longer Gaussian [e.g., Lee and Jokipii, 1975], yet $\theta_{s c}$ can be calculated as the equivalent $1 / e$ angular half-width of the radiation. Generalizing previous PWE derivations to include finite $f_{p o} / f$, the result is

$$
\begin{aligned}
\theta_{s c}^{2}(z)=\pi^{-2} & \left(4 \pi^{2} r_{e}^{2} \int d z \frac{C_{N}^{2}(z) \lambda^{\alpha}}{\left(1-f_{p 0}(z)^{2} / f^{2}\right)}\right. \\
& \left.\frac{\Gamma(2-\alpha / 2)}{\Gamma(\alpha / 2)} \frac{2^{2-\alpha}}{(\alpha-2)}\right)^{2 /(\alpha-2)}
\end{aligned}
$$

where $\Gamma(p)$ denotes the incomplete Gamma function and $r_{e}$ is the classical electron radius. Inserting (4) into (5), the integrand is proportional to $R^{-4.05}$ for $f \gg f_{p}$; i.e., the rate of scattering decreases with increasing $R$ for a given $f$. At 1 AU (5) predicts scattered source sizes in good accord with the ISEE observations quoted above. Table 1 shows the results of extrapolating this model into the outer heliosphere using the same path length and observation position as for the Gaussian model. Once again the calculated effects of scattering are very substantial. In fact, $\theta_{s c}$ is predicted to be much larger than Gurnett et al.'s [1993] observations indicate. This argues that scattering is plausibly very important, but also suggests that the level of density turbulence is smaller than in (4). The differences between the Gaussian and Kolmogorov predictions show the importance of properly modelling the turbulence. 
Table 1. Predicted and observed $\theta_{s c}(50 \mathrm{AU})$ in radians

\begin{tabular}{cccc}
\hline Model & $\begin{array}{c}\theta_{s c} \\
(2 \mathrm{kHz})\end{array}$ & $\begin{array}{c}\theta_{s c} \\
(3 \mathrm{kHz})\end{array}$ & $\begin{array}{c}\text { Observed } \theta_{s c} \\
(3 \mathrm{kHz})\end{array}$ \\
\hline Gaussian & 2.4 & 1.0 & 1.0 \\
Kolmogorov & 76 & 30 & 1.0 \\
\hline
\end{tabular}

\section{Predicted radial variations}

Motivated by the semi-quantitative success of the above calculations, and the observation of turbulent density spectra that are close to Kolmogorov at distances $\sim 0.3-10 \mathrm{AU}$, it is appropriate to provide more detailed predictions for $\theta_{s c}\left(R, f, f_{p 0}\right)$ using a Kolmogorov density spectrum with level $C_{N}^{2}(R)=3.9 \times$ $10^{15} w\left(R / R_{S}\right)^{-4}$ that is closely related to (4). The quantity $w$ describes the relative value of $C_{N}^{2}(R)$ relative to (4). The path length integral (5) is easily converted into an integral over $R$ for a radial path from the source to the observer. Writing $f_{p}(R)=f_{p 1} R^{-1}$ in steady-state, where $f_{p 1}$ is the plasma frequency at $1 \mathrm{AU}$ and $R$ is the heliocentric distance measured in $\mathrm{AU}$, the path length integral can be evaluated analytically:

$$
\theta_{s c}^{5 / 3}=B \lambda^{11 / 3} w\left[\frac{2}{A^{2} R}+\frac{1}{A^{3}} \log \left(\frac{R-A}{R+A}\right)\right]_{R_{\circ}}^{R_{0}}
$$

Here the observer is at $R_{O}$, the source is at $R_{\mathrm{s}}, A=$ $f_{p 1} / f$, and $B=7.0 \times 10^{-12}$. It may then be shown that

$$
\theta_{\mathrm{app}} \propto R_{O}^{-1.8} f^{-2.2} w^{0.6}
$$

when $R_{s} \gg R_{O}, f \gtrsim 2 f_{p 0}(z)$, and $\theta_{s c} \gg \theta_{\text {in }}$. Figure 1 shows the variations in $\theta_{\mathrm{app}}(R)$ predicted by (6) at $f=$ 2 and $3 \mathrm{kHz}$ for $w=1.0$ and 0.1 . Note the agreement with (7) at large $R$. At small $R \lesssim 2 f_{p 1} / f$, however, $\theta_{s c}$ increases faster than $R_{o}^{-1.8}$ due to the $1-f_{p 0}^{2}(z) / f^{2}$ term in (5). Comparing Figure 1 with Table 1 it is

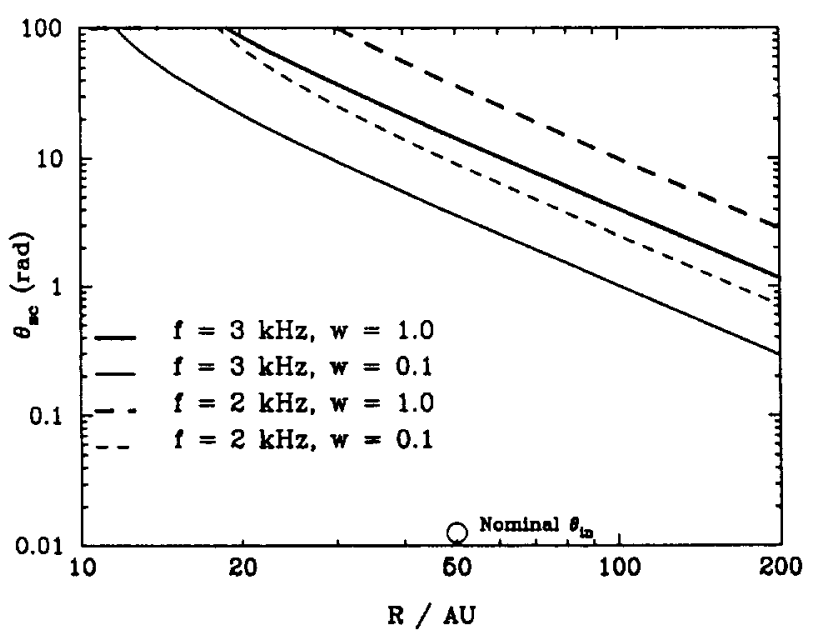

Figure 1. Predictions for $\theta_{s c}\left(R_{O}\right)$ versus $R_{O}$.

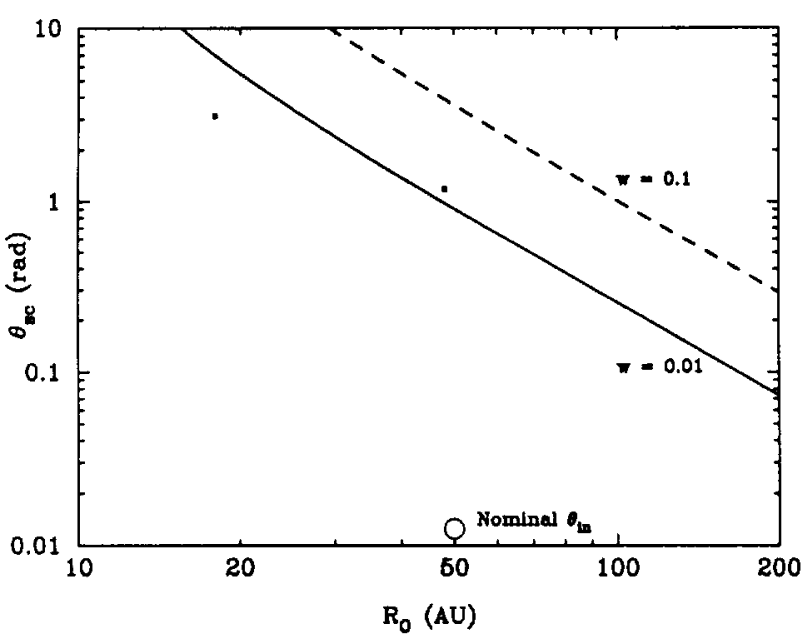

Figure 2. Comparison of $\theta_{s c}\left(R_{O}\right)$ versus $R_{O}$ with data.

clear that scattering is predicted to cause major angular broadening of the $2-3 \mathrm{kHz}$ radiation source.

If scattering is important and the source is in the outer heliosphere, then one important prediction of (3)$(7)$ is that $\theta_{\mathrm{BC}}$ will decrease with increasing $R$. Contrarily, for radiation produced in the inner heliosphere at $R_{1}, \theta_{\mathrm{sc}}(R)$ will increase with increasing (decreasing) $R$ for $R>R_{1}\left(R<R_{1}\right)$. If scattering is important, then, radial trends in $\theta_{\text {app }}(R)$ can establish if the radiation is produced in the outer or inner heliosphere. (It may be necessary to also consider radial variations in $\theta_{\text {in }}$, which decreases as the source becomes more distant.)

These predictions are, of course, preliminary due to their reliance on a turbulence model extrapolated from within $1 \mathrm{AU}$ and the inclusion of scattering in the solar wind alone. Future refinements should include new turbulence models and (most likely) scattering in the heliosheath. Observational tests of predictions for $\theta_{\text {app }}$ should then constrain the characteristics of the density turbulence between the source and observer and, ideally, the location and nature of the source.

\section{First evidence for radial trends in the roll modulation data?}

Two sets of published roll modulation data exist for radiation near $3 \mathrm{kHz}$ and apparent source sizes can be extracted therefrom. Voyager 1 was at $R \sim 18 \mathrm{AU}$ for Kurth's [1988] data and at $R \sim 45-48$ AU for Gurnett et al.'s [1993] data. Kurth's [1988] data show a very weak modulation pattern (the peak and trough values differ by $\sim 1 \%$ ) whose asymmetry casts some doubt on its interpretation. It is thus appropriate to take $\theta_{\text {app }}=\pi \mathrm{rad}$ as an approximate lower limit. In comparison, near $48 \mathrm{AU}$ Gurnett et al.'s [1993] Figure 2 implies significantly larger modulation and therefore smaller $\theta_{\text {app }}$. Taking the source to have constant intensity per unit solid angle, standard calculations imply that an upper limit on $\theta_{\text {app }}$ is $\sim 1.0 \mathrm{rad}$. (The source's 
elevation above the spacecraft's roll plane affects $\theta_{\mathrm{app}}$, with larger elevations implying smaller $\theta_{\mathrm{app}}$ for the same modulation pattern. Here the source is assumed to lie in the roll plane.) The two data sets thus show $\theta_{\mathrm{app}}$ decreasing by a factor $\gtrsim 3$ from $18 \mathrm{AU}$ to $48 \mathrm{AU}$. Assuming small intrinsic source variations, as suggested by the close similarities between the 1983/84 and 1992/93 events [e.g., Gurnett et al., 1993], the data imply that $\theta_{\text {app }}(R)$ decreases with increasing $R$, as predicted by (6) - (7) and Figure 1. Recent data at $R \gtrsim 50 \mathrm{AU}$ [Gurnett and Kurth, 1994] have even larger modulations than the $48 \mathrm{AU}$ data, thereby further suggesting that $\theta_{\text {app }}$ increases with $R$. The lack of roll modulation near 2 $\mathrm{kHz}$ is not inconsistent with scattering theory.

Figure 2 compares the prediction (6) for $f=3 \mathrm{kHz}$ and $w=10^{-2}$ and $10^{-1}$ with the observed $\theta_{\text {app. }}$. Reasonable agreement is apparent for $w=10^{-2}$. This implies three results. First, scattering is indeed quantitatively important in determining the observable characteristics of the $2-3 \mathrm{kHz}$ radiation. Second, the observed variations in $\theta_{\text {app }}(R)$ are consistent with the source being in the outer heliosphere but inconsistent with the source being inner heliospheric (e.g., Jupiter). (See Gurnett and Kurth [1994] for other arguments against a Jovian source.) Third, either the density turbulence is weaker by a factor $\sim 10^{2}$ than the model (4) or else other aspects of the turbulence model or the distance to the source need revision in order to obtain semiquantitative agreement between data and theory. In either case, these results argue for further work on scattering theory for the $2-3 \mathrm{kHz}$ radiation and on MHD turbulence in the outer heliosphere.

\section{Conclusions}

Calculations are presented of angular scattering of radio waves by density irregularities, with application to the $2-3 \mathrm{kHz}$ emissions observed by the Voyager spacecraft outside $\sim 10 \mathrm{AU}$. Two models for the turbulence, both of which are physically plausible and accurately account for scattering near and well inside $1 \mathrm{AU}$, are extrapolated into the outer heliosphere. For nominal path lengths $\sim 50 \mathrm{AU}$ and both turbulence models the calculations predict significant angular broadening. Indeed, scattering alone can account for the source sizes observed. Preliminary predictions for radial variations in the scattered source size $\theta_{s c}$ are presented, based on extrapolation of the Armstrong-Woo Kolmogorov model well beyond $1 \mathrm{AU}$ and a path solely in the solar wind. Good agreement between the predictions and data are obtained if the turbulence level is $\sim 100$ times smaller than the Armstrong/Woo model. One prediction is that the apparent source size of an outer heliospheric source should decrease as $R$ increases. Voyager roll observations at $R=18$ and $48 \mathrm{AU}$ are qualitatively consistent with this prediction, arguing that scattering is important and that the radiation is outer heliospheric and not from Jupiter. Additional qualitative evidence for scattering is provided by the lack of modulation near $2 \mathrm{kHz}$ and the variability in apparent source direction. While intrinsic changes in the source's size, direction, and in- tensity are possible and even likely, these calculations and the analogy with type III bursts etc. demonstrate the great plausibility of scattering changing the apparent size and other characteristics of the source. Scattering should be carefully considered when interpreting and modeling the $2-3 \mathrm{kHz}$ radiation.

Acknowledgments. I thank S.R. Spangler, J.W. Armstrong, M.J. Reiner, G.P. Zank, and D.A. Roberts for helpful suggestions. NASA grant 42415 supported this work.

\section{References}

Armstrong, J.W., and R. Woo, Jet Propulsion IOM 333180-070, 1980.

Cairns, I.H., W.S. Kurth, and D.A. Gurnett, Outer heliospheric radio emissions 2. Foreshock source models, J. Geophys. Res., 97, 6245, 1992.

Gurnett, D.A., W.S. Kurth, S.C. Allendorf, and R.L. Poynter, Radio emission from the heliopause triggered by an interplanetary shock, Science, 262, 199, 1993.

Gurnett, D.A., and W. S. Kurth, Evidence that Jupiter is not the source of the $2-3 \mathrm{kHz}$ heliospheric radiation, Geophys. Res. Lett., 21, 1571, 1994.

Hollweg, J.V., Angular broadening of radio sources by solar wind turbulence, J. Geophys. Res., 75, 3715, 1970.

Kurth, W.S., The low frequency interplanetary radio emission: evidence of the solar wind-interstellar wind interaction?, in Proc. of the Sixth International Solar Wind Conference, Vol. II, Eds. V.J. Pizzo, T.E. Holzer, and D.G. Sime, pg. 667, 1988.

Kurth, W.S., D.A. Gurnett, F.L. Scarf, and R.L. Poynter, Detection of a radio emission at $3 \mathrm{kHz}$ in the outer heliosphere, Nature, 312, 27, 1984.

Lacombe, C., C.C. Harvey, S. Hoang, A. Mangeney, J.-L. Steinberg, and D. Burgess, ISEE observations of radiation at twice the solar wind plasma frequency, Ann. Geophys., $6,113,1988$.

Lee, L.C., and J.R. Jokipii, Strong scintillations in astrophysics. I. The Markov approximation, its validity and application to angular broadening, Astrophys. J., 196, $695,1975$.

Rickett, B.J., Interstellar scattering and scintillation of radio waves, Ann. Rev. Astron. Astrophys., 15, 479, 1977.

Spangler, S.R., and J.W. Armstrong, Low-frequency angular broadening and diffuse interstellar plasma turbulence, in Low Frequency Astrophysics from Space, Eds. N.E. Kassim and K.W. Weiler, pg. 156, 1990.

Steinberg, J.-L., S. Hoang, and G.A. Dulk, Evidence of scattering effects on the sizes of interplanetary type III radio bursts, Astron. Astrophys., 150, 205, 1985.

Steinberg, J.T., S. Hoang, and C. Lacombe, Propagation of terrestrial kilometric radiation through the magnetosheath, Ann. Geophys., 7, 151, 1989.

Steinberg, J.-L., M. Aubier-Giraud, Y. Leblanc, and A. Boischot, Scattering, absorption, and refraction of solar radio bursts, Astron. Astrophys., 10, 362, 1971.

Tatarski, V.I., The Effects of the Turbulence Atmosphere on Wave Propagation, Israel Program for Scientifc Translation, 1971.

Iver H. Cairns, Department of Physics and Astronomy, University of lowa, lowa City, IA 52242.

(received January 20, 1995;

revised August 11, 1995;

accepted October 3, 1995.) 